\title{
Large screening of CA-MRSA among Staphylococcus aureus colonizing healthy young children living in two areas (urban and rural) of Portugal
}

\author{
Débora A Tavares ${ }^{1}$, Raquel Sá-Leão 1,2, Maria Miragaia ${ }^{1}$ and Hermínia de Lencastre*1,3
}

\begin{abstract}
Background: The incidence of pediatric infections due to community-associated methicillin-resistant Staphylococcus aureus (CA-MRSA), including children with no identifiable risk factors, has increased worldwide in the last decade. This suggests that healthy children may constitute a reservoir of MRSA in the community. In this study, nested within a larger one on nasopharyngeal ecology, we aimed to: (i) evaluate the prevalence of MRSA colonizing young children in Portugal; and (ii) compare results with those obtained in a study conducted a decade ago, when this prevalence was $<0.5 \%$.

Methods: In the years 2006, 2007, and 2009, nasopharyngeal samples were obtained from 2,100 children aged up to 6 years attending day-care centers. S. aureus were isolated by routine procedures and strains were tested for susceptibility against a panel of 12 antimicrobial agents. MRSA isolates were further characterized by Smal-PFGE profiling, MLST, spa typing, SCCmec typing, and presence of virulence factors.

Results: Seventeen percent of the children carried S. aureus. Among the 365 isolates, non-susceptibility rates were $88 \%$ to penicillin, $14 \%$ to erythromycin, $6 \%$ to clindamycin, $2 \%$ to tetracycline, and $<1 \%$ to oxacillin, rifampicin, ciprofloxacin, and SXT. Three MRSA strains were isolated. These had properties of CA-MRSA, such as low-level resistance to oxacillin and limited resistance to non-beta-lactams. Two CA-MRSA were related to USA700 (ST72-IV): one was ST72-IVc, spa type t148; the other was ST939-IVa (ST939 is a single locus variant (SLV) of ST72), spa type t324. The third strain was related to USA300 (ST8-IV) being characterized by ST931 (SLV of ST8)-VI, spa type t008. The three MRSA strains were PVL-negative, but all carried LukE-LukD leukocidin, hemolysins gamma, gamma variant and beta, and staphylococcal enterotoxin sel.

Conclusions: Our results, based on analysis of S. aureus isolated from nasopharyngeal samples, suggest that in Portugal the prevalence of CA-MRSA carriage in healthy young children remains extremely low favoring the exclusion of this group as a reservoir of such isolates.
\end{abstract}

\section{Background}

Staphylococcus aureus colonizes asymptomatically mainly the anterior nares of humans. It is also a frequent cause of clinically important infections [1]. Methicillinresistant S. aureus (MRSA) emerged in the 1960s, shortly after the introduction of methicillin in clinical practice, through the acquisition of the $m e c A$ gene. The gene $m e c A$

* Correspondence: lencash@mail.rockefeller.edu

1 Laboratory of Molecular Genetics, Instituto de Tecnologia Química e Biológica (ITQB), Universidade Nova de Lisboa (UNL), Oeiras, Portugal

Full list of author information is available at the end of the article encodes for a low affinity penicillin binding protein, PBP2A, and is carried by the staphylococcal chromosomal cassette mec (SCCmec) [2,3]. Molecular characterization of a large collection of isolates from different geographic regions showed that SCCmec has been acquired in more than one event by already epidemic genetic backgrounds, giving rise to five main pandemic clones that have spread worldwide [4,5]. Nowadays, MRSA prevalence in hospitals can range from less than 
5\% in countries from Northern Europe and The Netherlands, to more than $70 \%$ in Japan and Hong Kong $[6,7]$.

Three decades after the emergence of MRSA, when infection control policies aimed to decrease the prevalence of MRSA in hospitals of several countries were being implemented MRSA changed its epidemiology. From a restricted hospital-associated pathogen, MRSA made its way into the community [8]. During the last decade, an increasing incidence of infections in the community due to MRSA has been reported [9]. In particular, pediatric infections due to community-associated MRSA (CA-MRSA) among children with no identifiable predisposing risks for health-care associated infection have been documented worldwide [10-12]. A major and unsettled issue in the worldwide emergence of CA-MRSA is the source of such isolates in the community. The abovementioned observations led some authors to propose that healthy children might constitute an asymptomatic reservoir of CA-MRSA [13].

While numerous surveillance programs have been producing information on resistance patterns and molecular types of S. aureus in hospitals, relatively little information is available on strains of this species that colonize healthy human populations. Information on this issue has become particularly important with the worldwide emergence of drug resistant strains - particularly epidemic MRSA lineages - outside hospitals, in the "healthy" human community.

In Portugal, the prevalence of nosocomial MRSA varies widely between hospitals but, overall, remains among the highest $(53 \%)$ in Europe $[6,14]$. Moreover, the prevalence of MRSA causing skin and soft tissue infections (SSTIs) in Portugal was the highest in Europe between 1998 and 2004 [15]. However, the carriage and infection rates of MRSA in the community remain mostly unknown.

An earlier study performed in Portugal on the composition of $S$. aureus flora colonizing healthy human populations provided a striking demonstration on the virtual absence of MRSA in healthy humans in the late 1990-s [16], i.e., at a time when MRSA strains were already contributing to over $50 \%$ of $S$. aureus infections in Portuguese hospitals. Indeed, of five MRSA out of $285 \mathrm{~S}$. aureus isolates recovered from 2,111 children in that study, three were related to nosocomial clones. The other two were of ST82 and had low-level resistance to oxacillin [16].

In this report we examined a large collection of nasopharyngeal samples from healthy children taken between 2006 and 2009 for S. aureus and for the presence and/or frequency of MRSA strains. The comparison of this time period with the previous study encompasses the worldwide emergence of CA-MRSA and thus should provide an unbiased sample to monitor whether significant changes in the representation of MRSA in this population have occurred. To achieve such goal, we enrolled over 2,000 children between 2006 and 2009. Participants were enrolled in two areas: Oeiras (urban municipality) and Montemor-o-Novo (rural municipality). To our best knowledge, this is one of the largest studies conducted so far worldwide aiming to identify CA-MRSA in this risk group.

\section{Methods}

In the winter months of January-March of 2006, 2007, and 2009, as part of an ongoing study on colonization patterns by Streptococcus pneumoniae (R. Sá-Leão, unpublished data, [17] nasopharyngeal swabs were collected from children aged 4 months to 6 years attending DCCs in the urban area of Oeiras. In 2009, the study was extended to the rural area of Montemor-o-Novo. The DCCs were selected to cover the spectrum of social backgrounds found in both areas. Approval for the study was obtained from the Ministry of Education and Directors of DCCs. Parents or guardians gave written informed consent, and a questionnaire was filled in with information on illnesses, antibiotic consumption, and attendance to hospitals within the six months preceding sampling.

In each year, a single sample was collected from each child. Overall, 2,100 samples were obtained: 571 in 2006, 538 in 2007, and 991 in 2009 (611 from Oeiras and 380 from Montemor-o-Novo).

Nasopharyngeal swabs were taken as described [17], and inoculated within four hours onto mannitol salt agar (Difco, Detroit, MI). Plates were incubated in aerobic conditions for $18 \mathrm{~h}-48 \mathrm{~h}$ at $37^{\circ} \mathrm{C}$. Single morphologically distinct mannitol-positive colonies were isolated and pure cultures were tested for coagulase production by using Staphytect Plus (Oxoid, Hampshire, England), according to the manufacturers' instructions. Cultures that were mannitol-positive and coagulase-negative were also tested with Coagulase Plasma Rabbit (Becton, Dickinson and Company, Sparks, MD), according to the manufacturers' instructions. Cultures that were mannitolpositive and coagulase-positive were considered to be $S$. aureus.

Antimicrobial susceptibility to penicillin, oxacillin, erythromycin, clindamycin, tetracycline, vancomycin, ciprofloxacin, rifampicin, linezolid, quinupristin/dalfopristin, gentamicin, and trimethropim/sulphamethoxazole (SXT) was tested by disk diffusion, according to the Clinical Laboratory Standards Institute recommendations and definitions for all S. aureus isolates[18]. Disks were purchased from Oxoid, Hampshire, England. Isolates were classified as either susceptible or non-susceptible, the latter classification including isolates with both intermediate and resistant phenotypes.

All isolates showing an inhibition zone smaller than 20 $\mathrm{mm}$ surrounding the oxacillin disk were screened by PCR 
for the presence of the $m e c A$ gene using primers mecAP4 and mecAP7 [19]. MICs to oxacillin were determined for all isolates and to vancomycin for MRSA isolates only; MICs were determined by Etest (AB Biodisk, Slona, Sweden).

MRSA isolates were further characterized by pulsedfield gel electrophoresis (PFGE) [20], spa typing [21], multilocus sequence typing (MLST) [22], and SCCmec typing $[4,23-25]$. The presence of genes coding for virulence factors Panton-Valentine leukocidin $(p v l)$, LukELukD leukocidin (lukED), class F leukocidin (lukM), staphylococcal enterotoxins (sea-e, seg-j, sep, sel), toxic shock syndrome toxin (tsst), exfoliative toxins (eta, etb, $e t d$ ), and hemolysins (gamma $[h l g]$, gamma variant [hlgv], and beta $[h l b]$ ) was screened by multiplex PCR reactions as previously described [26-28].

The two MRSA isolates recovered from children in 1997 (during the previous study) that were not associated with nosocomial clones were also characterized by spa typing and SCCmec typing, and the presence for virulence genes was screened, as above [16].

\section{Results}

\section{S. aureus carriage}

A total of 2,100 samples were obtained. The mean age of the participants was 3.5 years and $52.4 \%$ were male. $S$. aureus were isolated from $17.4 \%$ of the 2,100 samples, ranging from $13.2 \%$ to $21.6 \%$ depending on the sampling year and origin of isolates (Table 1). In line with previous observations, [29-31] carriage of S. aureus was significantly associated with age ranging from $6.3 \%$ among children aged less than two years and steadily increasing up to $27.5 \%$ among those aged six years (data not shown).

\section{Antimicrobial susceptibility}

Among the 365 S. aureus, $11.5 \%$ were susceptible to all antibiotics tested and the frequency did not vary significantly within the four collections.

Non-susceptibility rates were $88.5 \%$ to penicillin, $14.0 \%$ to erythromycin, $5.5 \%$ to clindamycin, $2.2 \%$ to tetracy- cline, and $<1 \%$ to oxacillin (three isolates), rifampicin (three isolates), ciprofloxacin (two isolates), and SXT (single isolate). All isolates were susceptible to vancomycin, linezolid, quinupristin/dalfopristin, and gentamicin. Antibiotic resistant rates to individual antibiotics were not significantly different within the four collections.

\section{MRSA carriage}

The three oxacillin-resistant isolates were confirmed to carry the mecA gene and to have low-level resistance to this antibiotic (Table 2). The MRSA isolates were recovered from girls (two aged 3 , and one aged 5 years) attending different DCCs in Oeiras in 2006. One girl had received three or more courses of antibiotics in the six months preceding sampling. None had attended a hospital in the previous six months. Other risk factors associated with carriage of CA-MRSA were not investigated.

\section{Molecular characterization of MRSA}

Molecular characterization was done for the three MRSA isolates identified in this study as well as for the two ST82 identified in the 1996-1998 study [16]. Two MRSA strains isolated in 2006 were, by PFGE, related to USA700 (ST72-IV): one strain was of ST939-IVa, spa type t324; the other was of ST72-IVc, spa type t148 (Figure 1, Table 2). ST939 is a single-locus variant (SLV) of ST72. The spa types are single repeat variants among each other and both differ in three repeats from the spa type of USA700 prototype strain (t126).

The third MRSA strain isolated in 2006 was, by PFGE, related to USA300 (ST8-IV). This isolate was of ST931VI, spa type t008. ST931 is a SLV of ST8 and spa type t008 has been associated with USA300 representatives. Of notice, this strain had a MIC to oxacillin of $2 \mathrm{mg} / \mathrm{L}$, below the indicative breakpoint of $4 \mathrm{mg} / \mathrm{L}$.

The three MRSA strains were PVL-negative, but all carried five other virulence genes: $l u k E D, h l g, h l g v, h l b$, and sel (Table 2).

The characterization of the two ST82 isolates described in the 1996-1998 study confirmed they were related to each other but not identical: strain DCC1028 was of spa

Table 1: Study collection and distribution of S. aureus isolates

\begin{tabular}{|c|c|c|c|c|c|}
\hline \multirow[t]{2}{*}{ Year } & \multirow[t]{2}{*}{ Geographic origin } & \multirow[t]{2}{*}{ Day care centers } & \multicolumn{3}{|c|}{ No. (\%) } \\
\hline & & & Nasopharyngeal samples & S. aureus & MRSA \\
\hline 2006 & Oeiras & 11 & 571 & $92(16.1)$ & $3(0.53)$ \\
\hline 2007 & Oeiras & 11 & 538 & $71(13.2)$ & 0 \\
\hline 2009 & Oeiras & 9 & 611 & $120(19.6)$ & 0 \\
\hline \multirow[t]{2}{*}{2009} & Montemor-o-Novo & 16 & 380 & $82(21.6)$ & 0 \\
\hline & & Total & 2,100 & $365(17.4)$ & $3(0.14)$ \\
\hline
\end{tabular}


Table 2: Characterization of MRSA strains

\begin{tabular}{|c|c|c|c|c|c|c|c|c|c|c|}
\hline \multirow[t]{2}{*}{ Strain ID } & \multirow[t]{2}{*}{ Isolation year } & \multirow{2}{*}{$\begin{array}{l}\text { Antibiotypea } \\
\text { (resistant to) }\end{array}$} & \multirow{2}{*}{$\begin{array}{c}\text { Oxacillin MIC } \\
\text { (mg/L) }\end{array}$} & \multirow[t]{2}{*}{ MLST } & \multirow[t]{2}{*}{ PFGE } & \multicolumn{2}{|c|}{ spa type } & \multirow[t]{2}{*}{ SCC mec type } & \multirow{2}{*}{ Virulence factorsb } & \multirow[t]{2}{*}{ Representative of } \\
\hline & & & & & & RIDOM & eGenomics & & & \\
\hline DCC5292 & 2006 & PEN, OXA & 24 & 939 & $A_{1}$ & $\mathrm{t} 324$ & $\begin{array}{c}451 \\
\text { (UJGGMDMGGM) }\end{array}$ & IVa & lukED, hlg, hlgv, hlb, sel & USA700 \\
\hline DCC5504 & 2006 & PEN, OXA & 2 & 931 & B & t008 & $\begin{array}{c}1049 \\
\text { (YHGFMBOBLO) }\end{array}$ & VI & lukED, hlg, hlgv, hlb, sel & USA300 \\
\hline DCC5723 & 2006 & PEN, OXA, ERY, CLI & 8 & 72 & $\mathrm{~A}_{2}$ & $\mathrm{t} 148$ & $\begin{array}{c}193 \\
\text { (UJGFGMDMGGM) }\end{array}$ & IVc & lukED, hlg, hlgv, hlb, sel & USA700 \\
\hline DCC1028 & 1997 & PEN, OXA, ERY & 12 & 82 & $C_{1}$ & $\mathrm{t} 186$ & $\begin{array}{c}9 \\
\text { (UGFMEEBBPB) }\end{array}$ & IVa & lukED, hlgv, hlb, sel, eta & \\
\hline DCC1076 & 1997 & PEN, OXA, ERY, GEN & 24 & 82 & $C_{2}$ & $\mathrm{t} 3221$ & $\begin{array}{c}1190 \\
\text { (UGEBBPB) }\end{array}$ & IVc & lukED, sep, hlg, hlgv, sel & \\
\hline
\end{tabular}

aPEN, penicillin; OXA, oxacillin; ERY, erythromycin; CLI, clindamycin; GEN, gentamicin

bVirulence factors tested: Panton-Valentine leukocidin (pvl), LukE-LukD leukocidin (lukED), class F leukocidin (lukM), staphylococcal enterotoxins (sea-e, seg-j, sel, sep), toxic shock syndrome toxin (tsst), exfoliative toxins (eta, etb, etd), and hemolysins (gamma [hlg], gamma variant [hlgv], and beta [hlb]) 


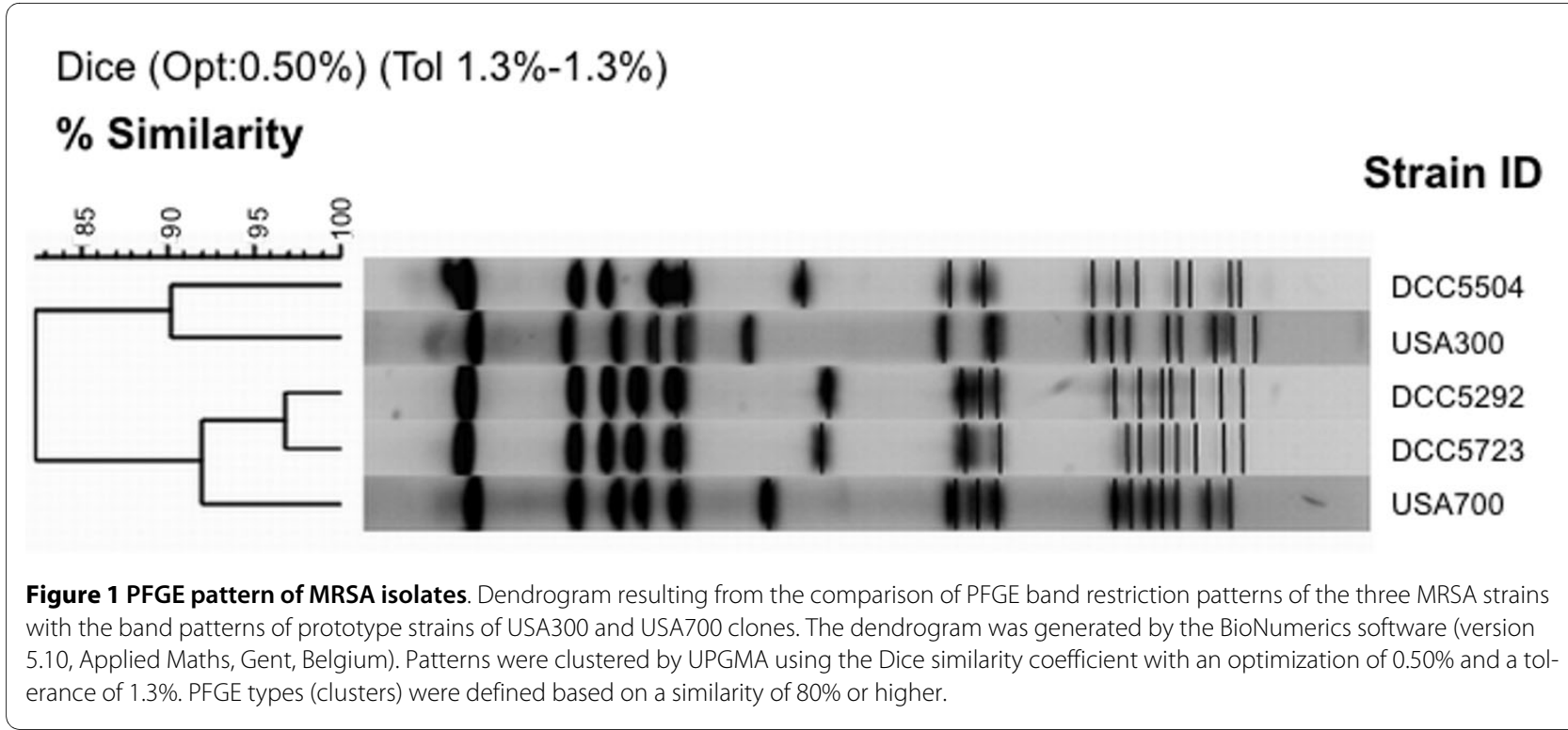

type t186 and carried SCCmecIVa and the genes lukED, $h l g v$, hlb, sel, and eta; strain DCC1076 was of spa type $\mathrm{t} 3221$ and carried SCCmecIVc and the genes lukED, hlg, $h l b$, sel, and sep. Both were PVL-negative. The spa types differed among each other in an insertion/deletion of three repeats (Table 2).

\section{Discussion}

During the last ten years we have been following the impact of antibiotic use and the introduction of the pneumococcal conjugate vaccine on the bacterial flora colonizing the nasopharynx of preschool children [17,32]. Although the nasopharyngeal sampling in these studies was designed to recover primarily the major microbial colonizers of the nasopharyngeal space, such as Streptococcus pneumoniae, Haemophilus influenzae, and Moraxella catarrhalis, the method also picks up a sample of other natural inhabitants of the upper respiratory tract - such as S. aureus. Thus, nasopharyngeal samples recovered from children in our surveillance program could also serve as a valuable source of information on possible changes that may have occurred in the composition of the natural flora of important human opportunistic pathogens, namely $S$. aureus.

Previous reports of CA-MRSA infections among children with no health-care associated risk factors provided evidence of a hospital-independent community reservoir [10-12,33]. In addition, other recent studies have identified risk factors for carriage and transmission of CAMRSA, and, consequently, populations at higher risk of CA-MRSA infections due to their life styles. Due to crowded conditions, intensive exposure to antimicrobial agents, and close contact, DCC attendees have also been considered a high-risk population for carriage of MRSA
[8]. Combined with an increasing incidence of infections in children with no predisposing risks factors, this population has been pointed as a possible community reservoir of CA-MRSA. In fact, recent studies from Greece and Japan reported rates of MRSA nasal carriage of 3.3\% and $3.7 \%$, respectively, corresponding to $5.5 \%$ and $9.1 \%$ of MRSA among total S. aureus isolates [13,34]. In Nashville, TN, and Taiwan MRSA nasal carriage rates have reached $7 \%$ to $9 \%$ of the sampled population (corresponding to $25-30 \%$ of MRSA among all S. aureus isolates) $[29,35]$. Finally, studies from Brazil and Illinois, IL, reported rates of MRSA nasopharyngeal carriage of 1.0\% and $1.7 \%$, respectively, corresponding to $7.5 \%$ and $9.3 \%$ of MRSA among total S. aureus isolates [36,37].

In the current study conducted among healthy children living in the Oeiras and Montemor-o-Novo municipalities of Portugal, we sampled a large group of children attending DCCs. As in the first study, nasopharyngeal MRSA colonization was found to be very low $(0.24 \%$ in 1996-1998 and 0.13\% in 2006-2009) [16]. Although we sampled the nasopharynx, which is not the preferred niche for S. aureus colonization, the proportion of MRSA among total $S$. aureus isolates was still very low: $1.77 \%$ in 1996-1998 and $0.82 \%$ in 2006-2009. This was a surprisingly low rate, in light of findings from other countries and knowledge of high prevalence of HA-MRSA in Portugal. However, one of the geographical areas studied is an urban area densely populated where use of antibiotics is intense and transmission of potential bacterial pathogens among children is known to be high and has been well documented [17]. In addition, in contrast to other European countries, in Portugal over $75 \%$ of pre-school children are attending DCCs [17], often since the early age of 4-5 months, five days a week, eight hours a day. 
Thus, our results may well represent the Portuguese reality.

Another recent study by Lamaro-Cardoso and coworkers also found a low prevalence (1.2\%) of MRSA among DCC attendees in Brazil. Excluding the fact that the annual median household income of this population was below the Brazilian poverty line, only 4 of 14 MRSA isolates were not associated to health-care settings, and all had been recovered from children with at least one risk factor [38].

The three MRSA isolates recovered in the present study had properties of typical CA-MRSA such as low-level resistance to oxacillin and limited resistance to non- $\beta$ lactams [9]. Furthermore, by molecular typing they were identified as related to CA-MRSA clones USA300, a major epidemic CA-MRSA clone in the USA that is also spreading in Europe and USA700, a clone that has been associated to community and health-care settings [39,40]. The MRSA strain related to USA300 carried SCCmec type VI, which was first described in a nosocomial MRSA clone identified in Portugal [41]. The two ST82 strains found a decade ago also showed low-level resistance to oxacillin and limited resistance to non- $\beta$-lactams. In addition, they carried SCCmec IV, also characteristic of CA-MRSA. Of interest, the strains isolated in 2006 were unrelated to these ones. However, MRSA strains of ST88IV (ST88 is a SLV of ST82) have been isolated in Portugal and China in the hospital setting, and in Nigeria and Switzerland in the community [42-46]. The emergence of a USA300 related clone among healthy children attending DCC is worrisome given its acknowledged high capacity of dissemination that can be rapidly amplified by the high rates of child-to-child transmission occurring in DCCs.

This study has some limitations. First, we sampled the nasopharynx, which is not the preferential niche of $S$. aureus and thus we probably underestimated the true prevalence of MRSA. In particular, we did not sample other sites of colonization such as anterior nares, throat, groin, and rectum [47]. Secondly, the study included only children aged up to six years who are less prone to be colonized by $S$. aureus than older children. Thirdly, we focused mainly in one urban region, even though a rural region was also studied in 2009. Although similar results were found over time and in both geographic locations, it remains unknown whether the observations made are representative of Portugal. Regardless of these limitations, we consider that this study contains valuable information on the evolution of MRSA carriage in asymptomatic children.

\section{Conclusions}

Our results, based on the analysis of S. aureus isolated from nasopharyngeal samples, suggest that in Portugal the prevalence of CA-MRSA carriage in healthy young children remains extremely low favoring the exclusion of this group as a reservoir of such isolates. Given the current concern about CA-MRSA, the observed emergence of a USA300 related clone in this study, and the high prevalence of MRSA causing SSTIs in Portugal [15], an extension of these studies to other risk groups and continuous surveillance is mandatory.

\section{Competing interests \\ The authors declare that they have no competing interests.}

\section{Authors' contributions}

DAT performed experimental work and drafted the manuscript. RSL, MM, and $\mathrm{HML}$ designed the study. RSL helped to draft the manuscript. MM and $\mathrm{HML}$ critically revised the manuscript. All authors have read and approved the final manuscript.

\section{Acknowledgements}

This work was supported by Fundação para a Ciência e Tecnologia (grants number PTDC/SAU-ESA/65048/2006 and PTDC/BIA-MIC/64010/2006); Fundação Calouste Gulbenkian (grant number P-99911); and the European Union (projects TROCAR, grant number FP7-HEALTH-2007-B 223031, and CONCORD, grant number FP7-HEALTH-2007-B 222718).

We are grateful to Alexander Tomasz for comments and criticisms that contributed to improve the manuscript. We thank Sónia Nunes, Nelson Frazão, Carina Valente, Alexandra Simões, Inês Crisóstomo, and Ana Tavares for participating in studies that led to the isolation of the Staphylococcus aureus strains. This publication made use of the Multi Locus Sequence Typing website http:// www.mlst.net at Imperial College London developed by David Aanensen and Man-Suen Chan and funded by the Wellcome Trust. We are grateful to Barry N. Kreiswirth and Steven Naidich for the spa type assignments through the egenomics website http://epigene.egenomics.com

\section{Author Details}

'Laboratory of Molecular Genetics, Instituto de Tecnologia Química e Biológica (ITQB), Universidade Nova de Lisboa (UNL), Oeiras, Portugal, ${ }^{2}$ Centro de Matemática e Aplicações Fundamentais (CMAF), Universidade de Lisboa, Lisboa, Portugal and ${ }^{3}$ Laboratory of Microbiology, The Rockefeller University, New York, NY, USA

Received: 1 February 2010 Accepted: 3 May 2010

Published: 3 May 2010

\section{References}

1. Wertheim HF, Melles DC, Vos MC, van Leeuwen W, van Belkum A, Verbrugh HA, Nouwen $\mathrm{J}$ : The role of nasal carriage in Staphylococcus aureus infections. Lancet Infect Dis 2005, 5(12):751-762.

2. Hartman BJ, Tomasz A: Low-affinity penicillin-binding protein associated with beta-lactam resistance in Staphylococcus aureus. J Bacteriol 1984, 158(2):513-516.

3. Katayama $Y$, Ito T, Hiramatsu K: A new class of genetic element, staphylococcus cassette chromosome mec, encodes methicillin resistance in Staphylococcus aureus. Antimicrob Agents Chemother 2000, 44(6):1549-1555.

4. Oliveira DC, Tomasz A, de Lencastre $\mathrm{H}$ : Secrets of success of a human pathogen: molecular evolution of pandemic clones of meticillinresistant Staphylococcus aureus. Lancet Infect Dis 2002, 2:180-189.

5. Enright MC, Robinson DA, Randle G, Feil EJ, Grundmann H, Spratt BG: The evolutionary history of methicillin-resistant Staphylococcus aureus (MRSA). Proc Natl Acad Sci USA 2002, 99(11):7687-7692.

6. EARSS annual report 2008 [http://www.rivm.nl/earss/Images/ EARSS\%202008 final tcm61-65020.pdf

7. Diekema DJ, Pfaller MA, Schmitz FJ, Smayevsky J, Bell J, Jones RN, Beach M: Survey of infections due to Staphylococcus species: frequency of occurrence and antimicrobial susceptibility of isolates collected in the United States, Canada, Latin America, Europe, and the Western Pacific region for the SENTRY Antimicrobial Surveillance Program, 1997-1999. Clin Infect Dis 2001, 32(Suppl 2):S114-132. 
8. de Lencastre H, Oliveira D, Tomasz A: Antibiotic resistant Staphylococcus aureus: a paradigm of adaptive power. Curr Opin Microbiol 2007 10(5):428-435

9. Chambers HF: The changing epidemiology of Staphylococcus aureus? Emerg Infect Dis 2001, 7(2):178-182.

10. Paintsil E: Pediatric community-acquired methicillin-resistant Staphylococcus aureus infection and colonization: trends and management. Curr Opin Pediatr 2007, 19(1):75-82.

11. O'Brien FG, Pearman JW, Gracey M, Riley TV, Grubb WB: Community strain of methicillin-resistant Staphylococcus aureus involved in a hospital outbreak. J Clin Microbiol 1999, 37(9):2858-2862.

12. CDC: From the Centers for Disease Control and Prevention. Four pediatric deaths from community-acquired methicillin-resistant Staphylococcus aureus-Minnesota and North Dakota, 1997-1999. JAMA 1999, 282(12):1123-1125.

13. Sdougkos G, Chini V, Papanastasiou DA, Christodoulou G, Stamatakis E, Vris A, Christodoulidi I, Protopapadakis G, Spiliopoulou I: Communityassociated Staphylococcus aureus infections and nasal carriage among children: molecular microbial data and clinical characteristics. Clin Microbiol Infect 2008, 14(11):995-1001.

14. Melo-Cristino J: Antimicrobial resistance in staphylococci and enterococci in 10 Portuguese hospitals in 1996 and 1997. POSGAR. Portuguese Study Group of Antimicrobial Resistance. Microb Drug Resist 1998, 4(4):319-324

15. Moet GJ, Jones RN, Biedenbach DJ, Stilwell MG, Fritsche TR: Contemporary causes of skin and soft tissue infections in North America, Latin America, and Europe: report from the SENTRY Antimicrobial Surveillance Program (1998-2004). Diagn Microbiol Infect Dis 2007, 57(1):7-13.

16. Sá-Leão R, Sanches IS, Couto I, Alves CR, de Lencastre H: Low prevalence of methicillin-resistant strains among Staphylococcus aureus colonizing young and healthy members of the community in Portugal. Microb Drug Resist 2001, 7(3):237-245

17. Sá-Leão R, Nunes S, Brito-Avô A, Frazão N, Simões AS, Crisóstomo Ml Paulo AC, Saldanha J, Santos-Sanches I, de Lencastre H: Changes in pneumococcal serotypes and antibiotypes carried by vaccinated and unvaccinated day-care centre attendees in Portugal, a country with widespread use of the seven-valent pneumococcal conjugate vaccine. Clin Microbiol Infect 2009, 15(11):1002-1007.

18. CLSI: Performance standards for antimicrobial disk susceptibility tests; approved standard, 10th edn. Wayne, PA: CLSI publication M02-A10. Clinical and Laboratory Standards Institute; 2009.

19. Oliveira DC, de Lencastre H: Multiplex PCR strategy for rapid identification of structural types and variants of the mec element in methicillin-resistant Staphylococcus aureus. Antimicrob Agents Chemother 2002, 46(7):2155-2161.

20. Chung M, de Lencastre H, Matthews P, Tomasz A, Adamsson I, Aires de Sousa M, Camou T, Cocuzza C, Corso A, Couto I, et al:: Molecular typing of methicillin-resistant Staphylococcus aureus by pulsed-field gel electrophoresis: comparison of results obtained in a multilaboratory effort using identical protocols and MRSA strains. Microb Drug Resist 2000, 6(3):189-198.

21. Aires-de-Sousa M, Boye K, de Lencastre H, Deplano A, Enright MC, Etienne J, Friedrich A, Harmsen D, Holmes A, Huijsdens XW, et al:: High interlaboratory reproducibility of DNA sequence-based typing of bacteria in a multicenter study. J Clin Microbiol 2006, 44(2):619-621.

22. Enright MC, Day NP, Davies CE, Peacock SJ, Spratt BG: Multilocus sequence typing for characterization of methicillin-resistant and methicillin-susceptible clones of Staphylococcus aureus. J Clin Microbiol 2000, 38(3):1008-1015.

23. Oliveira DC, Tomasz A, de Lencastre H: Secrets of success of a human pathogen: molecular evolution of pandemic clones of meticillinresistant Staphylococcus aureus. Lancet Infect Dis 2002, 2(3):180-189.

24. Milheiriço C, Oliveira DC, de Lencastre H: Update to the multiplex PCR strategy for assignment of mec element types in Staphylococcus aureus. Antimicrob Agents Chemother 2007, 51(9):3374-3377.

25. Milheiriço C, Oliveira DC, de Lencastre H: Multiplex PCR strategy for subtyping the staphylococcal cassette chromosome mec type IV in methicillin-resistant Staphylococcus aureus: 'SCCmec IV multiplex'. J Antimicrob Chemother 2007, 60(1):42-48.

26. Vandenesch F, Naimi T, Enright MC, Lina G, Nimmo GR, Heffernan $H$, Liassine N, Bes M, Greenland T, Reverdy ME, et al:: Community-acquired methicillin-resistant Staphylococcus aureus carrying Panton-Valentine leukocidin genes: worldwide emergence. Emerg Infect Dis 2003, 9(8):978-984.

27. Monday SR, Bohach GA: Use of multiplex PCR to detect classical and newly described pyrogenic toxin genes in staphylococcal isolates. $J$ Clin Microbiol 1999, 37(10):3411-3414.

28. Jarraud S, Mougel C, Thioulouse J, Lina G, Meugnier H, Forey F, Nesme X, Etienne J, Vandenesch F: Relationships between Staphylococcus aureus genetic background, virulence factors, agr groups (alleles), and human disease. Infect Immun 2002, 70(2):631-641

29. Creech CB, Kernodle DS, Alsentzer A, Wilson C, Edwards KM: Increasing rates of nasal carriage of methicillin-resistant Staphylococcus aureus in healthy children. Pediatr Infect Dis J 2005, 24(7):617-621.

30. Fritz SA, Garbutt J, Elward A, Shannon W, Storch GA: Prevalence of and risk factors for community-acquired methicillin-resistant and methicillin-sensitive Staphylococcus aureus colonization in children seen in a practice-based research network. Pediatrics 2008, 121(6):1090-1098

31. Masuda K, Masuda R, Nishi J, Tokuda K, Yoshinaga M, Miyata K: Incidences of nasopharyngeal colonization of respiratory bacterial pathogens in Japanese children attending day-care centers. Pediatr Int 2002, 44(4):376-380.

32. De Lencastre H, Kristinsson KG, Brito-Avô A, Sanches IS, Sá-Leão R, Saldanha J, Sigvaldadottir E, Karlsson S, Oliveira D, Mato R, et al.: Carriage of respiratory tract pathogens and molecular epidemiology of Streptococcus pneumoniae colonization in healthy children attending day care centers in Lisbon, Portugal. Microb Drug Resist 1999, 5(1):19-29.

33. Carleton HA, Diep BA, Charlebois ED, Sensabaugh GF, PerdreauRemington F: Community-adapted methicillin-resistant Staphylococcus aureus (MRSA): population dynamics of an expanding community reservoir of MRSA. J Infect Dis 2004, 190(10):1730-1738.

34. Ozaki K, Takano M, Higuchi W, Takano T, Yabe S, Nitahara Y, Nishiyama A, Yamamoto T: Genotypes, intrafamilial transmission, and virulence potential of nasal methicillin-resistant Staphylococcus aureus from children in the community. J Infect Chemother 2009, 15(2):84-91.

35. Huang YC, Hwang KP, Chen PY, Chen CJ, Lin TY: Prevalence of methicillin-resistant Staphylococcus aureus nasal colonization among Taiwanese children in 2005 and 2006. J Clin Microbiol 2007, 45(12):3992-3995

36. Lamaro-Cardoso J, Castanheira M, de Oliveira RM, e Silva SA, Pignatari AC, Mendes RE, Pimenta FC, Andrade AL: Carriage of methicillin-resistant Staphylococcus aureus in children in Brazil. Diagn Microbiol Infect Dis 2007, 57(4):467-470

37. Cheng Immergluck L, Kanungo S, Schwartz A, McIntyre A, Schreckenberger PC, Diaz PS: Prevalence of Streptococcus pneumoniae and Staphylococcus aureus nasopharyngeal colonization in healthy children in the United States. Epidemiol Infect 2004, 132(2):159-166.

38. Lamaro-Cardoso J, de Lencastre H, Kipnis A, Pimenta FC, Oliveira LS, Oliveira RM, Nouer SS, Aires-de-Sousa M, Milheirico C, Andrade AL: Molecular epidemiology and risk factors for nasal carriage of Staphylococcus aureus and methicillin-resistant $S$. aureus in infants attending day care centers in Brazil. J Clin Microbio/ 2009, 47(12):3991-3997.

39. McDougal LK, Steward CD, Killgore GE, Chaitram JM, McAllister SK Tenover FC: Pulsed-field gel electrophoresis typing of oxacillin-resistant Staphylococcus aureus isolates from the United States: establishing a national database. J Clin Microbiol 2003, 41(11):5113-5120

40. Diep BA, Gill SR, Chang RF, Phan TH, Chen JH, Davidson MG, Lin F, Lin J, Carleton HA, Mongodin EF, et al: Complete genome sequence of USA300, an epidemic clone of community-acquired meticillin-resistant Staphylococcus aureus. Lancet 2006, 367(9512):731-739.

41. Oliveira DC, Milheiriço C, de Lencastre $\mathrm{H}$ : Redefining a structural variant of staphylococcal cassette chromosome mec, SCCmec type VI. Antimicrob Agents Chemother 2006, 50(10):3457-3459.

42. Zhang W, Shen X, Zhang H, Wang C, Deng Q, Liu L, Yang Y: Molecular epidemiological analysis of methicillin-resistant Staphylococcus aureus isolates from Chinese pediatric patients. Eur J Clin Microbiol Infect Dis 2009, 28(7):861-864

43. Liu Y, Wang H, Du N, Shen E, Chen H, Niu J, Ye H, Chen M: Molecular evidence for spread of two major methicillin-resistant Staphylococcus aureus clones with a unique geographic distribution in Chinese hospitals. Antimicrob Agents Chemother 2009, 53(2):512-518. 
44. Harbarth S, Francois P, Shrenzel J, Fankhauser-Rodriguez C, Hugonnet S, Koessler T, Huyghe A, Pittet D: Community-associated methicillinresistant Staphylococcus aureus, Switzerland. Emerg Infect Dis 2005, 11(6):962-965.

45. Ghebremedhin B, Olugbosi MO, Raji AM, Layer F, Bakare RA, Konig B, Konig W: Emergence of a community-associated methicillin-resistant Staphylococcus aureus strain with a unique resistance profile in Southwest Nigeria. J Clin Microbiol 2009, 47(9):2975-2980.

46. Aires-de-Sousa M, Correia B, de Lencastre $\mathrm{H}$ : Changing patterns in frequency of recovery of five methicillin-resistant Staphylococcus aureus clones in Portuguese hospitals: surveillance over a 16-year period. J Clin Microbio/ 2008, 46(9):2912-2917.

47. Yang ES, Tan J, Eells S, Rieg G, Tagudar G, Miller LG: Body site colonization in patients with community-associated methicillin-resistant Staphylococcus aureus and other types of S. aureus skin infections. Clin Microbiol Infect 2010, 16:425-431.

\section{Pre-publication history}

The pre-publication history for this paper can be accessed here: http://www.biomedcentral.com/1471-2334/10/110/prepub

doi: $10.1186 / 1471-2334-10-110$

Cite this article as: Tavares et al., Large screening of CA-MRSA among Staphylococcus aureus colonizing healthy young children living in two areas (urban and rural) of Portugal BMC Infectious Diseases 2010, 10:110

\section{Submit your next manuscript to BioMed Central} and take full advantage of:

- Convenient online submission

- Thorough peer review

- No space constraints or color figure charges

- Immediate publication on acceptance

- Inclusion in PubMed, CAS, Scopus and Google Scholar

- Research which is freely available for redistribution

Submit your manuscript at www.biomedcentral.com/submit
C) Biomed Central 